\title{
Use of Mandibulo-Maxillary Fixation Screws for the Treatment of Maxillofacial Fractures
}

\section{Sanvritti Manjrekar ${ }^{*}$ and Apoorva Joshi}

Cactus Communications Inc, Austin, TX, United States

*Corresponding author: Sanvritti Manjrekar, BDS, MS, Senior Scientific Writer, Cactus Communications Inc, Austin, TX, United States, Tel: 6178930033; Fax: 6178930033; E-mail: sanvrittimanjrekar92@gmail.com

Received date: November 15, 2017; Accepted date: December 05, 2017; Published date: December 12, 2017

Copyright: (C) 2017 Manjrekar S, et al. This is an open-access article distributed under the terms of the Creative Commons Attribution License, which permits unrestricted use, distribution, and reproduction in any medium, provided the original author and source are credited.

\begin{abstract}
Maxillomandibular fractures can be described as a complete or incomplete break causing discontinuity of the bone tissues in the maxillary or mandibular structures resulting from the application of an excessive force. The intimate relationship between maxillofacial structures with the oral cavity, nasal cavity, orbits, and adjacent cranial structures make it a functionally and cosmetically important structure.Most etiological causes of these fractures are: road accidents, contact sport injuries, assaults, falls and other industrial accidents. Several epidemiological studies have shown that these fractures tend to occur in males between the ages of 16-40.

Fractures of these bones are often considered life threatening due to potential airway obstruction and cause interference with speech, masticatory functions, and aesthetic appearance Therefore, it is necessary for the systematic and timely repair of these fractures to correct deformity and restore normal function of the masticatory structures.

MMF has long been used as a fixation technique for reduction of fractures by wiring or banding the maxilla and mandible to reduce the fracture. MMF screws have time and again proven themselves as the a revolutionary technique. Therefore, it is necessary to understand the use of MMF screws in its techniques for further use in maxillo mandibular fracture.
\end{abstract}

Keywords Maxillomandibular fractures; Fracture; Bone

\section{Introduction}

Maxillomandibular fractures can be described as a complete or incomplete break causing discontinuity of the bone tissues in the maxillary or mandibular structures resulting from the application of an excessive force. The intimate relationship between maxillofacial structures with the oral cavity, nasal cavity, orbits, and adjacent cranial structures make it a functionally and cosmetically important structure [1].

Most etiological causes of these fractures are: road accidents, contact sport injuries, assaults, falls and other industrial accidents. Several epidemiological studies have shown that these fractures tend to occur in males between the ages of 16-40 [2].

Fractures of these bones are often considered life threatening due to potential airway obstruction and cause interference with speech, masticatory functions, and aesthetic appearance Therefore, it is necessary for the systematic and timely repair of these fractures to correct deformity and restore normal function of the masticatory structures.

In planning the systematic and timely reconstruction of the complex maxillofacial fractures, it is necessary to understand distinguished topographic areas of the face and the classification of these fractures.

The maxillofacial structures can be classified into:

\section{- The Mandible}

\section{Midface Fractures}

The anatomic region between the maxillary teeth and the infraorbital margin is referred to as the midface. Le Fort classified the midface fractures into three levels along commonly occurring lines in 1901. This classification was based on his cadaver experiments and has been one of the most acceptable classification over the last century.

\section{Fracture Classification by Le Fort (1901)}

- Le Fort I: There is disarticulation of tooth-bearing part of the maxilla at the level of the floor of nose and maxillary sinuses (low level fracture of the maxilla).

- Le Fort II: There is disarticulation of the maxilla and the nasal skeleton (pyramidal fracture of the maxilla).

- Le Fort III: There is disarticulation of the maxilla, the zygomatic bone, and the nasal skeleton (high level fracture of the midface).

- The Midface 

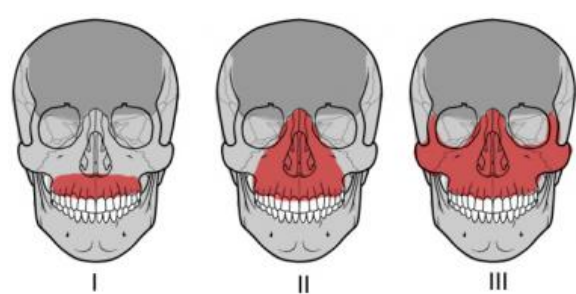

Figure 1: Le fort Fractures.

However, in modern clinical practice, most fractures are complex in nature, resulting in comminuted fractures with major bone loss and do not occur along the anatomical weak points of the midface (Figure 1).

Therefore, the necessity of a more current treatment-related classification has been recognized.

\section{Modified Le Fort classification: Marciani 1993 (NOE, ZMC)}

- Le Fort I - low maxillary fracture Ia-low maxillary fracture/ multiple segment

- LeFort II-pyramidal fractures IIa-pyramidal \& nasal fracture IIbpyramidal \& NOE fracture

- LeFort III-craniofacial disjunction IIIa- +nasal fracture IIIb+NasoOrbitoEthmoid fracture

- LeFort IV-LeFort II or III fracture \& cranial base fracture

- IVa- +supraorbital rim fracture

- IVb- +anterior cranial fossa \& supraorbital rim fracture

\section{Fractures of the Mandible}

- Median fractures

- Paramedian fractures

- Mandibular angle fractures

- Fractures of the ramus

- Condylar neck fracture

- Intra-capsular fractures

\section{CLASSIFICATION OF MANDIBULAR FRACTURES}

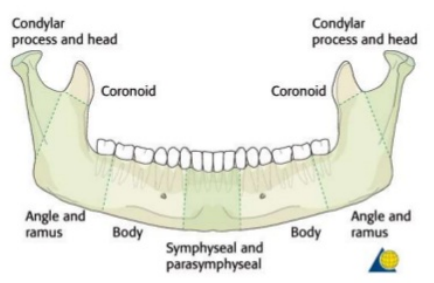

Figure 2: Classification of mandibular fractures.

\section{Clinical Assessment of Maxillofacial Fractures}

\section{Extra oral examination}

Midface fractures often present themselves with swelling, facial asymmetry, bruising, circumorbital ecchymosis (racoons eye) periorbital edema and subconjunctival hemorrhage. Mandibular fractures often have characteristic facial asymmetry, trismus, marked step offs along the symphysis and angle on palpation (Figure 2).

\section{Intraoral examination}

Maxillofacial fractures present themselves with disturbed occlusion, midline diastema, hematoma in the palate, edema, or ecchymosis in the floor of the mouth and fractured cusps of teeth (Figure 3).

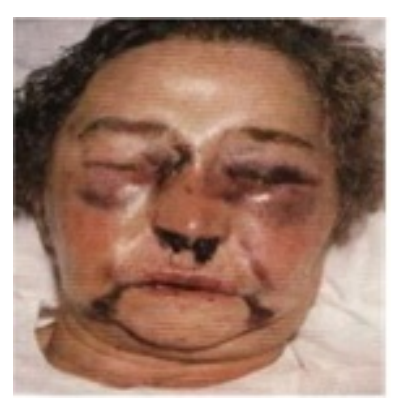

Figure 3: Extraoral clinical findings of maxillofacial fractures.

\section{Treatment of Maxillofacial Fractures}

There are two important steps involved in treatment of fractures:

Reduction: It is the technique of manipulating the fractured segments into a desirable position. It can be open: involves surgical reduction or closed: non-surgical reduction.

Fixation: Immobilization of the fractured segments which aids in the reunion of the bony fragments without the formation of any undesirable callouses. 


\section{Mandibulo-maxillary fixation}

It is also commonly known as Intermaxillary fixation, a technique used to stabilize a fractured jaw, where the teeth are wired or banded together. It is regarded as a crucial step in the management of maxillofacial fractures as it secures the interrelationship of occlusal surfaces, an essential step in reduction of fractured fragments in both jaws (Figure 4) [3].

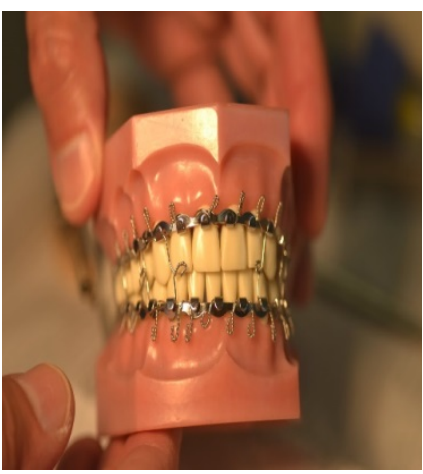

Figure 4: Mandibulo-Maxillary fixation.

\section{Traditional techniques}

Traditional techniques in dentate patients used devices such as arch bars, dental and interdental wiring, metallic and nonmetallic splints which were mounted upon the tooth to achieve intermaxillary fixation. In most of these techniques complex metallic framework was attached to the teeth. Interconnecting ligature wires/bands or elastics were passed between the dental arches serving as anchor points for immobilization of the jaws (Figures 5 and 6).

In patients with partial or complete edentulousness, "gunning" type splints were commonly used. Hooks were attached residual teeth in the oral cavity or wired circumferentially or would be directly screwed into alveolar processes, thereby creating an occlusal platform to apply the fixation ligatures [4].

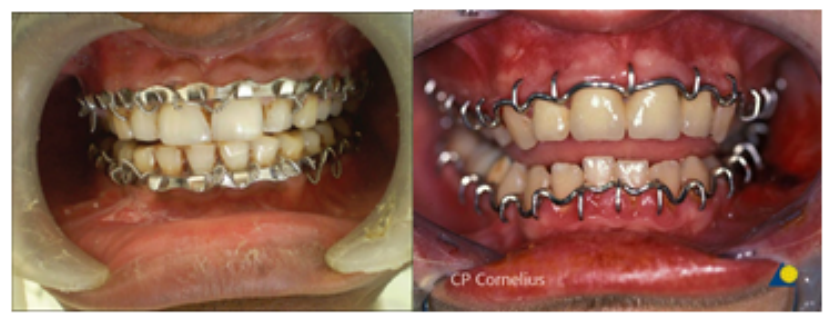

Figure 5: MMF using traditional arch bar wiring.

\section{Mandibulo Maxillary Fixation (MMF) Screws}

The introduction of MMF screws in 1989 have eliminated many of the above-mentioned techniques [5]. The screws are inserted into the alveolar process of the mandible and maxilla, providing anchor points for MMF linkage apparatus with specialized screw heads [3]. Most MMF's are used for a prolonged period for about 6 weeks of duration, which distinguishes them from temporary intraoperative fixation. This helps in achieving desired union of the fractured segments, occlusion and jaw movements.

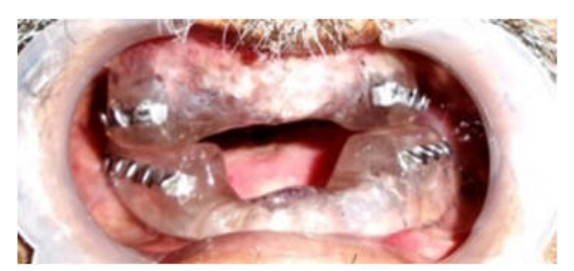

Figure 6: Traditional splinting technique in edentulous patients.

Conventional MMF screws were kept at $4-5 \mathrm{~mm}$ above the mucosa for subsequent wire splinting, they were wider in diameter and had limited grooves/flutes. They required surgical preparation of the site prior to insertion into the bone, thereby increasing the risk of damaging dental roots. The understanding of unique bone density of the maxilla and mandible led to designing self-drilling or self-tapping Maxillomandibular fixation screws. Often manufactured using stainless steel or unalloyed high-grade titanium, these screws have pointed tips, reduced thread diameter and are grooved with single, double, or triple straight or helical flutes, providing a reservoir for the bone debris and aiding faster bone formation. Self-drilling screws are coupled with hand held screwdrivers used in insertion providing necessary torque (Figure 7)

Most of the current screws are spool shaped and the central part of the screw head is perforated with one of two channels perpendicular to its axis aiding in the passage of ligature wires. Therefore, the wires will not slip over the screw heads. The top of the screw heads is also smoothly finished at the contact zone with the overlying buccal mucosa to out rule any ulcers or erosions.

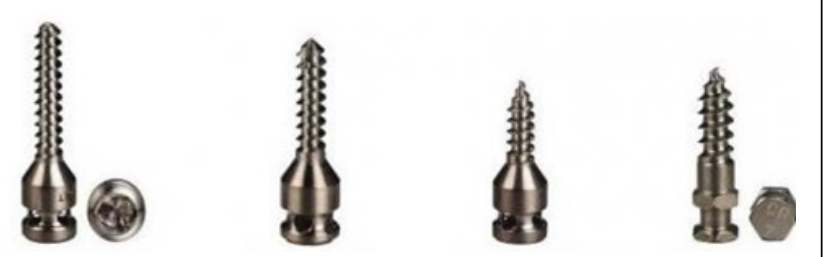

Figure 7: MMF Screws (Self-tapping).

\section{Screw Site selection}

Some of the considerations for site selection include:

- Fracture location

- Dentition

- Surgical exposure

- Quality of bone

Most preferred sites for maxillomandibular fixation screw placement post radiographic examinations such as panoramic x-rays, axial CT scans or digital volume tomography scans are the anterior vestibule and the anterolateral (canine and premolar regions) as they supply appropriate amount of bone depth and clearance from tooth roots along with easy accessibility. Use of these scans in pre-operative 
planning serves as a precision guiding point for the insertion of the screws without injuring the tooth roots, maxillary antrum, mandibular canal, or mental foramen.

Maxillomandibular Fixation Screw placement is avoided in the lateral surface of the maxillary base and the bone plates at midlevel height of mandibular body as these surfaces are constituted with thin bone and are in close proximity with the pneumatized maxillary sinus and the mandibular canal respectively. Placing maxillomandibular fixation screws closer to the coronal third of the roots serves as relative risk within the confines of upper and lower alveolar process as the anatomical structure of interdental septa converges towards the limbus and diverges with the circumference of adjacent tooth.

\section{Indications for MaxilloMandibular Fixation Screws}

- Stable occlusal platform to guide preinjury relationship when wire cerclages are placed.

- Simple fractures in fully dentate mandible or maxillary fractures.

- Fractures of alveolar process.

- Transverse palatal fractures by establishing side to side stability.

\section{Contraindications}

- Multiple fractures of the jaws with absence of opposing teeth due to lack of occlusal plane.

- Bone atrophy.

- Severe comminuted / pan facial fractures.

- Preinjury skeletal discrepancies.

- Congenital or acquired osteopathy's.

- Deciduous or early mixed dentition as the chances of damaging the tooth buds or developing roots is relatively high.

\section{Surgical Techniques}

Using radiological evidence, the Maxillomandibular Fixation screws can be inserted in several ways under either local or general anesthesia. The surgical site is prepared once the teeth and oral cavity are cleaned using disinfectant solution.

- Some of the most common insertion techniques are as follows:

- Perforation of closed mucosa or using an open approach after exposure of bone surface.

- Self-tapping after drilling pilot holes.

- Completely self-drilling.

- The gingival or vestibular mucosa can be perforated using a No. 11 blade scalpel or electrocautery needle.

- Self-tapping technique involves drilling full thickness pilot holes using a slow speed hand piece with copious irrigation at the mucogingival junction throughout all bony layers or after predrilling just the outer cortex. The burr is passed through the outer cortex, the medullary space and optionally into the lingual or palatal cortices, depending on whether a monocortical or bicortical placement is desired. The burr hole reduces the force needed for the insertion process.

- Completely self-drilling Maxillomandibular fixation screws do not require any preparation and can be driven into the bone by hand using coupled screwdriver and tools. Insertion starts with unengaged turns on the bony surface before the screw tip breaches the cortical shell and engages. After piercing the outer cortex, resistance drops abruptly which marks the entry into the spongy bone, then the screw is pulled into the bone by rotation resembling a corkscrew. Predrilling is often recommended if thickness of the bone cortex exceeds 2.5 to $3 \mathrm{~mm}$ (Gibbons, et al. 2003) [6].

- Once enough Maxillomandibular fixation screws have been inserted, then the occlusal relationships are precisely adjusted. This process may aide in further possible reduction of the bony fragments. The ends of the cerclage wire $(0.4$ or $0.5 \mathrm{~mm})$ are passed through the ligature holes in the screw heads and twisted under a constant pull with intermediate tugging motions in a vertical or diagonal direction resulting either in a straight or zig zag pattern until the wire loop is straight and fully tightened [3].

- Direct looping of ligature wires is possible when the opposing screws axes are oriented at divergent angles. Screw should preferably be backed up in desired position to make ligature holes easily accessible. At least two-thirds of the screw shaft should be inserted into the bone, provided there is sufficient bone thickness for substantially holding the ligature wires in place.

- Removal of Maxillomandibular fixation screws after completion of therapy is simply done by a screwdriver. After a long-term trans mucous placement, the screws can be taken out without local anesthesia in the clinic. The small wound heals within a few days. Screws buried under soft tissue, which may present itself as one of the few common problems associated with the use of Maxillomandibular fixation screws may be exposed under local anesthesia and removed (Figures 8 and 9) [7].

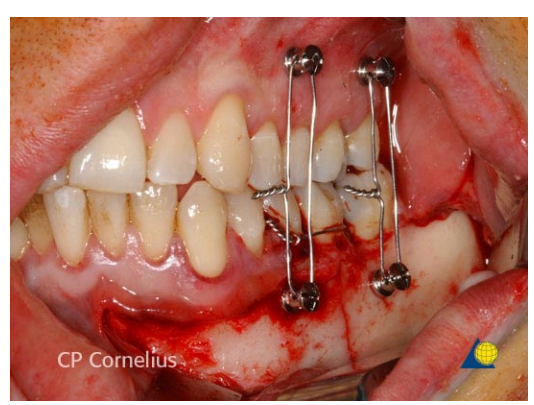

Figure 8: Maxillomandibular fixation Screws placed with vertical ligature wiring.

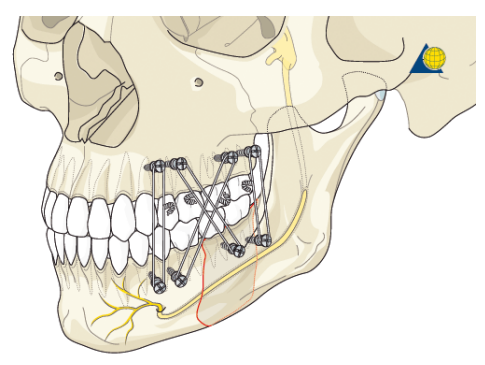

Figure 9: Maxillomandibular fixation Screws placed with zig zag pattern of ligature wiring. 


\section{Discussion}

\section{Advantages of maxillomandibular fixation}

The simplicity of technical handling of Maxillomandibular fixation screws provide improved abutments for jaw immobilization along with enhanced safety as fewer linkage is used. They can be used in several types of fractures, allow open reduction and are compatible in bone plating osteosynthesis. They are cost effective, easy to apply and do not require prolonged operating hours. Rate of glove perforations are diminished as it requires lesser intraoral manipulation using sharp stainless wire tips thereby decreasing blood borne virus transmissions such as HIV and Hepatitis. It is possible to use heavy traction elastics, orthodontic appliances, and high stability skeletal anchorage around the screws as there is more manipulation space in the oral cavity. Lesser appliances inside the oral cavity paves way to use of prosthodontic crowns, dentures, bridgework along with better infection control, oral hygiene maintenance and improved nutrition. They can be removed in the dental office painlessly and heal relatively quickly [8].

\section{Disadvantages of Maxillomandibular fixation}

Often, maxillomandibular screws have been associated with disadvantages such as iatrogenic damage to tooth roots, thereby causing tooth loss around the adjacent areas of screw placement. Most screws get covered by oral mucosa, which can be removed by a simple incision, and healing is prominent within two weeks of screw removal. Some studies have elicited other common complications such as periodontium loss, leading to tooth loss, screw sheer, root fracture, malocclusion, and ingested hardware [9].

\section{Conclusion}

Maxillomandibular fixation Screws have established themselves as a strong favorable method in the treatment of maxillomandibular fractures. This method may often be described as invasive, but is reliable, time saving, marginally painless as compared to traditional techniques and economic with relatively low contraindications and complications. Studies have shown decreased infection, lesions in the oral cavity, thereby providing increased comfort to the patient in the maintenance of oral higiene [3]. Reported risks and complications involving Maxillomandibular fixation screws such as dental root injuries, perforations into the foramina are minimal and can be overcome by better radiological examination and pre-operative planning.

With such high proven positive results, it is necessary to further research into the possibilities of the uses of Maxillomandibular fixation screws in more complex maxillofacial fractures. Improved biomechanical design of screws along with imaging techniques should help expand the horizons of Maxillomandibular fixation screws in oral and maxillofacial surgery.

\section{References}

1. Kühnel TS, Reichert TE (2015) Trauma of the midface. GMS Curr Top Otorhinolaryngology Head Neck Surg 14

2. Motamedi MHK, Dadgar E, Ebrahimi A, Shirani G, Haghighat A, et al. (2014) Pattern of maxillofacial fractures: A 5-year analysis of 8,818 patients. J Trauma Acute Care Surg, 77: 630-634.

3. Cornelius CP, Ehrenfeld M (2010) The Use of MMF Screws: Surgical Technique, Indications, Contraindications, and Common Problems in Review of the Literature. Craniomaxillofac Trauma Reconstr 3: 55-80.

4. Chaudhary, Z, Sharma R, Krishnan S (2014) Maxillo Mandibular Fixation in Edentulous Scenarios: Combined MMF Screws and Gunning Splints. J Maxillofac Oral Surg 13: 213-214.

5. Qureshi AA, Reddy UK, Warad NM, Badal S, Jamadar AA, et al. (2016) Intermaxillary fixation screws versus Erich arch bars in mandibular fractures: A comparative study and review of literature. Ann Maxillofac Surg 6: 24-30.

6. Gibbons AJ, Baden JM, Monaghan AM, Dhariwal DK, Hodder SC (2003) A Drill-free Bone Screw For Intermaxillary Fixation In Military Casualties. J R Army Med Corps, 149: 30-32.

7. Gordon KF, Reed JM, Anand VK (1995) Results of Intraoral Cortical Bone Screw Fixation Technique for Mandibular Fractures. Otolaryngol Head Neck Surg 113 248-252.

8. Pigadas N, Whitley SP, Roberts SA, McAlister K, Ameerally P, et al. (2017) A randomized controlled trial on cross-infection control in maxillofacial trauma surgery: A comparison of intermaxillary fixation techniques. Int J Oral Maxillofac Surg 37: 716-722.

9. Hashemi HM, Parhiz A (2011) Complications Using Intermaxillary Fixation Screws. J Oral Maxillofac Surg 69: 1411-1414. 